\title{
A VR-based CAD System
}

\author{
J.M. Zheng, K.W. Chan, I. Gibson
}

Department of Mechanical Engineering, The University of Hong Kong, Pokfulam Road, Hong Kong

Keywords (VR) Virtual reality, Geometric modelling, Computer graphics, CAD/CAM

\begin{abstract}
The performance of current Computer-Aided Design (CAD) systems is still far from the satisfaction of conceptual designers. This paper presents a VR-based CAD system for conceptual design by applying VR interfaces and geometric modelling techniques to improve the human computer interaction. The system uses an electronic data glove as an input device so that conceptual designers are allowed to use hand gestures to conduct various geometric shape operations instead of depending solely on keyboard and 2D mouse. We employ the 3D GUIs for enhancing the gesture interface. The implemented shape modelling techniques offered by the system include: destruction, construction, and techniques for freeform feature creation and modification. The destructive techniques allow the user to arbitrarily sculpt an existing part by means of sculpting tools. The constructive techniques allow the user to assemble an object by using a collection of feature objects, by either adding or subtracting feature objects from the model. The system also offers several novel methods for facilitating intuitive modification of freeform curves and surfaces.
\end{abstract}

\section{INTRODUCTION}

Current Computer-Aided Design (CAD) systems are not suitable for conceptual design because they have three significant drawbacks: i) designers are required to specify detailed geometry of the part in a very quantitative manner with tools that are more suitable to a draughtsman than a concept designer; ii) there is a lack of high-level shape operators for designing and modifying model shapes; and iii) the CAD interface is still mainly based on the use of $2 \mathrm{D}$ pointing and display devices. This makes the interface unnatural and non-intuitive.

In this paper, we use a CyberGlove, an electronic hand glove developed by Virtual Technology Inc., as an input device to develop a desktop CAD modelling system for facilitating conceptual design. Gestures are used to 
support intuitive user interface. To develop this gesture interface, we consider that conceptual designers should be given freedom to use different kinds of natural gestures to conduct various geometric shape operations instead of depending solely on keyboard and 2D mouse. For instance, designers can indicate objects or directions simply by pointing with their hand and fingers, and look at an object from different directions through grasping them and turning them into proper orientations and positions. The functional tools can be used for creating, cutting, and editing objects. We also employ 3D GUIs for enhancing the gesture interface. In the virtual environment, 3D menu and "virtual hand" are concurrently used. Various 3D cursors can be used to select menu or manipulate the object. The modelling methods used in the VR-based CAD system include feature based modelling techniques for constructing product model, such as constructive technique and destructive technique.

The VR-based CAD system consists of three parts: input, output and computation engine. The diagram of the system architecture is shown in Figure 1. As shown in Figure 1, the inputs include 2D mouse, keyboard, data glove input control, and position and orientation tracker. The glove input control allows the computer to determine the user's hand gesture and its physical position in 3D space.

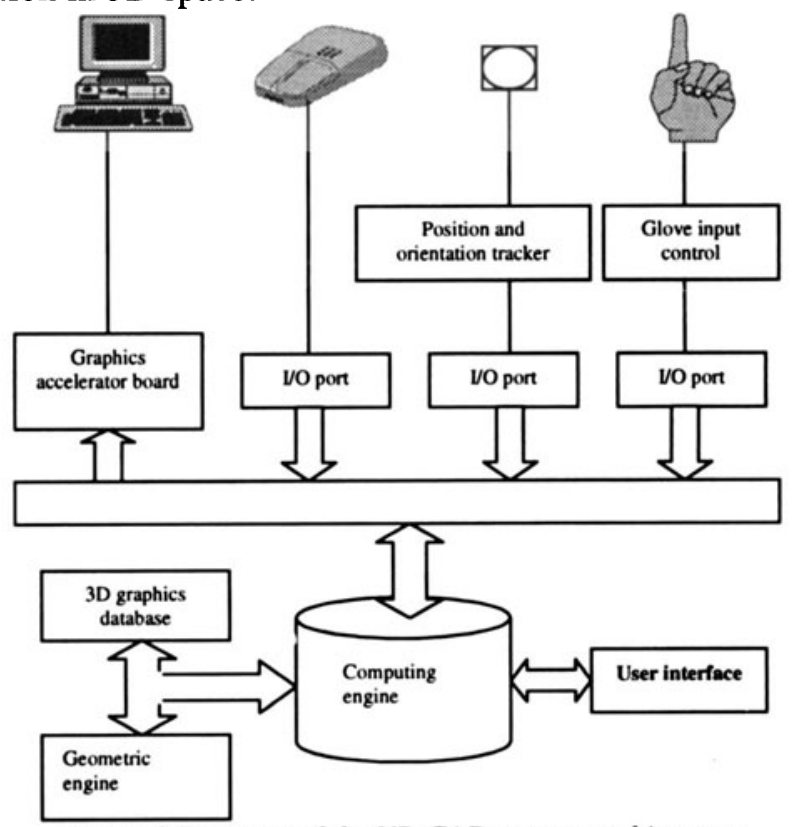

Figure 1 Diagram of the VR-CAD system architecture 


\section{RELATED WORKS}

There are many research activities on VR-based design systems. Based on the interactive ability that the designer can manipulate the product models, current VR-based product design systems can be classified into two main groups: enhanced visualization and VR-based CAD [1].

In the enhanced visualization group, the product part models that are previously created by traditional CAD modelling systems are imported into the VR-based environment through an appropriate transformation. Once the models of the product parts are imported into the virtual environment, 3D interactive devices, such as Dataglove, 3D mouse and 3D display monitor, can be used for the designer to operate the product model and navigate the product model in the virtual environment. Researchers at the University of North Carolina at Chapel Hill [2] presented a system called Immersive Simulation Animation And Construction (ISAAC) for the designer to interactively construct virtual worlds. The system allows the designer to position, orient and scale the pre-generated 3D models that are created by CAD modelling systems or 3D scanning devices in the virtual environment. Fraunhofer Institute for Computer Graphics, Darmstadt, Germany, developed a VR tool set, called Virtual Design II [19], to support the virtual product development applications. The system lets users import data from various sources, preprocess and enhance data, interact with and manipulate data in real time, and present the application using various audio-visual facilities. Other examples include the VENUS project [3] and research at Clemson University [4].

Different from the enhanced visualization group that is limited to just visualizing $\mathrm{CAD}$ models, the VR-based CAD systems allow the design activity to occur in the virtual environment. The designer can use $3 \mathrm{D}$ devices such as Dataglove, 3D mouse and 3D monitor such as Head Mounted Display (HMD) to create and modify the product models in the virtual environment. The advantage of the VR-based CAD is to provide a more intuitive $3 \mathrm{D}$ interface than traditional CAD systems and to offer enhanced tools for the designer to model product configuration. In addition, the VR-based CAD also supports alternative methods of user input, such as voice and gestures. Researchers at the Center for Design Research (CRD) at Stanford University carried out a number of projects on Human Computer Interfaces [5]. Other examples include the VR research at the I-CARVE $\mathrm{LAB}$ of the University of Wisconsin in Madison [1,6,7]. Researchers in the Brown University Graphics Group developed human-centered and interactive 3D virtual environments for modelling, scientific visualization, tele-collaboration, and interactive illustrations in a shared visual, spatial, and 
auditory environment $[8,9,10,11,12,13]$. The system can offer an intuitive user interface for the user, however, the modelling tools for mechanical design are still limited. The JDCAD system [14] used a pair of input devices and $3 \mathrm{D}$ interface menu to allow the designer to define objects. The Conceptual Design Space (CDS) [15], developed at Georgia Technical College, offers a real-time 3D immersive environment for 3D architectural design. The designer can use CDS to create conceptual building designs and modify them, add details, or create new designs, all are immersed in the virtual world. The System Research Laboratory of the Nippon Electronic Company developed a prototype CAD system for the designer to manipulate a CAD model by Dataglove [16]. The system can perform some simple shape manipulation functions but the accuracy achieved is not satisfactory for normal CAD applications.

\section{USER INTERFACE DESIGN}

\subsection{Guideline for user interface design}

Consistency: The consideration of consistency means that the interface should be consistent in its requirements for input and have consistent mechanisms for users to make any demands on the system. The consistent interface is one in which the interface elements are uniform and follow a few simple rules. The basic aim of consistency is to allow users to generalize knowledge about one aspect of the system to other aspects.

Feedback: Feedback refers to the process of sending back information to the user about what has been done. The consideration in feedback means that if the user has performed an action that triggered an internal system response or an important message is issued, the computer system should give an indication to show whether the message is accepted by the computer system. Therefore, the feedback can tell users that the requested operation has been completed and the new or modified display can explicitly show the results of the operation. For example, a selected object or menu command is highlighted so that the user can know that her/his actions have been accepted.

The desktop VR-based CAD system attempts to incorporate Windows's user interface elements, such as windows, icons, dialog box, and pull-down menus, so as to provide easy-understood feedback. The feedback involved in the VR-based CAD system can be divided into two aspects: object feedback and position feedback. The object feedback occurs when the user issues a command to select object. For example, when a command for selecting an object is issued, the requested object will be displayed in red color to 
indicate that the selection is accepted. A button (in which a gesture bitmap is embodied) is down to indicate that the corresponding gesture is recognized and accepted by the system.

Naturalness: Naturalness requires that the interface should not ask for redundant information from the user. It should require the minimum of user input and should provide an intuitive mechanism for the completion of the users' tasks.

Gestures are the main input mechanisms in the VR-based CAD system. It is important for the user interface to eliminate unnecessary memorization so that the users are comfortable to conduct various shape designs via gestures. In the VR-based CAD system, the suggestive gestures are displayed on the prompted dialog box shown on the left side of the screen. The suggestive gestures keep the request for information to the minimum and allow the user to choose different gestures to conduct commands or shape operations.

\subsection{Human computer interactive tasks in virtual environment}

Navigation: The navigation task provides the user the ability to navigate through the virtual space to view the generated scenes in the virtual space. The hand glove input device can provide a natural method, allowing the users to "physically" hold the object. The users can also grasp the object and dynamically rotate the object so as to allow the object to be viewed from different angles. While the user is navigating the virtual space, the visual output shows the updated 3D images. The user may repeatedly perform navigation operation in this navigation task until he/she satisfies the scene.

User commands: Although there is a different view on the use of user commands in virtual environment, we consider that the need for traditional type of user commands will not be completely eliminated in a virtual reality system because not all tasks are suited to direct manipulation or gestures.

In VR-based CAD system, menu-based commands may be viewed as a supplementary user interface for the virtual environment while the gestures are inadequate for the desired tasks. In order to allow the user to quickly learn the hierarchy tree, the menu items can be structured into groups. For example, the menus in the VR-based modelling system are grouped as the solid feature modelling menu and freeform feature modelling menus. The items in each menu can be grouped by their functions. Figure 2 shows the flow diagram for the user to navigate the hierarchical menu tree. The user first issues a gesture to choose a modelling method, then a 3D menu will be 
popped up and displayed in front of the user. Finally the user can use his/her hand to select a menu item from the menu. After the menu item selection is finished, the 3D menu will disappear from the screen.

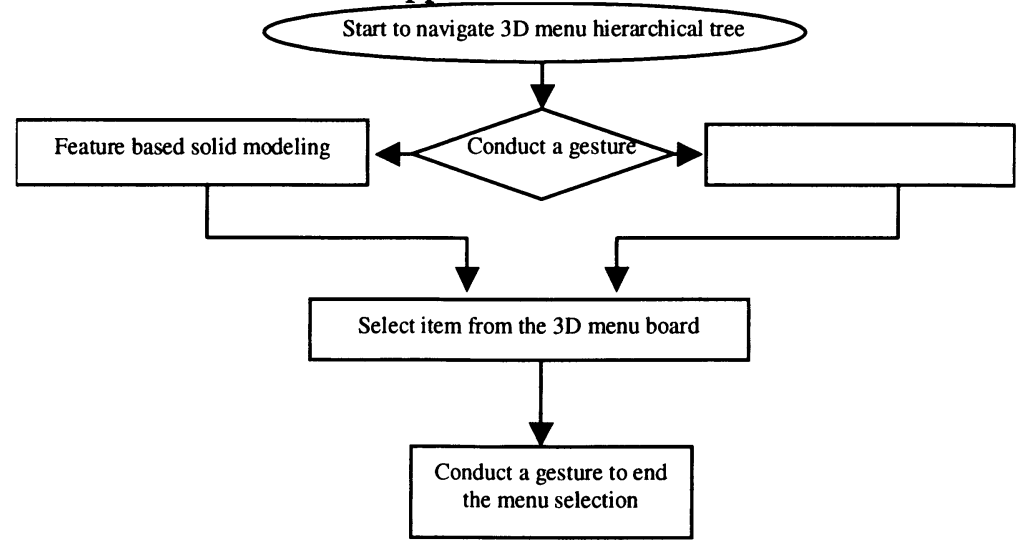

Figure 2 The flow diagram for the user to navigate the $3 D$ hierarchical tree

Object selection: The selection task is that of choosing an element from an object or menu item from the menu hierarchical tree. The intuitive and natural method for object selection in the VR-based CAD system should be to select an object when the user's hand comes into contact with it. The index finger is used to indicate an object to be selected. This method works well in the immersive VR system that can offer natural visual senses. In this situation, it is natural for user to use his/her hand in the same way that he/she would select an object in the physical world. However, this method may not work in current desktop virtual environment. Firstly, the hand motion may not be accurate enough to indicate some small object that needs to be selected. Secondly, the user has to navigate to the distant objects in order to select. Due to the display limitation of a desktop virtual environment, it may be difficult for the user to sense if she/he has touched the object. The raycasting technique can be used to solve this problem. In the VR-based CAD system, a ray is extended from the user's hand to intercept the desired object, and the first object intersected by the ray is selected. The visual viewer output of the system will check whether the user has indicated the object to be selected by highlighting the selected object.

Object manipulation: The object manipulation tasks in the VR-based CAD system can be cataloged into two groups. The first sub-category of manipulation tasks contains those actions which are symbolic or abstract in nature, such as loading files, selection of colors, and changes of system parameters. Those tasks that do not need 3D spatial input can be 
implemented by 2D mouse. The second category of manipulation tasks is object creation.

The object creation is the most important subcategory in object manipulation task. Figure 3 shows the steps for creating a cylinder. The user can select the cylinder item from the 3D menu and then a dialog box will pop up to guide the user to issue various gesture-based commands to conduct the object manipulation tasks. The user may use the index finger to indicate the location of the entity that is being created. The visual output feedback can also indicate whether the selected tasks have been accepted and processed. All object manipulation activities in the virtual environment are processed by hand gestures. The system also supports a mechanism for the user to specify accurate shape sizes. When all creation parameters are determined, the user can issue a gesture command to create the object.

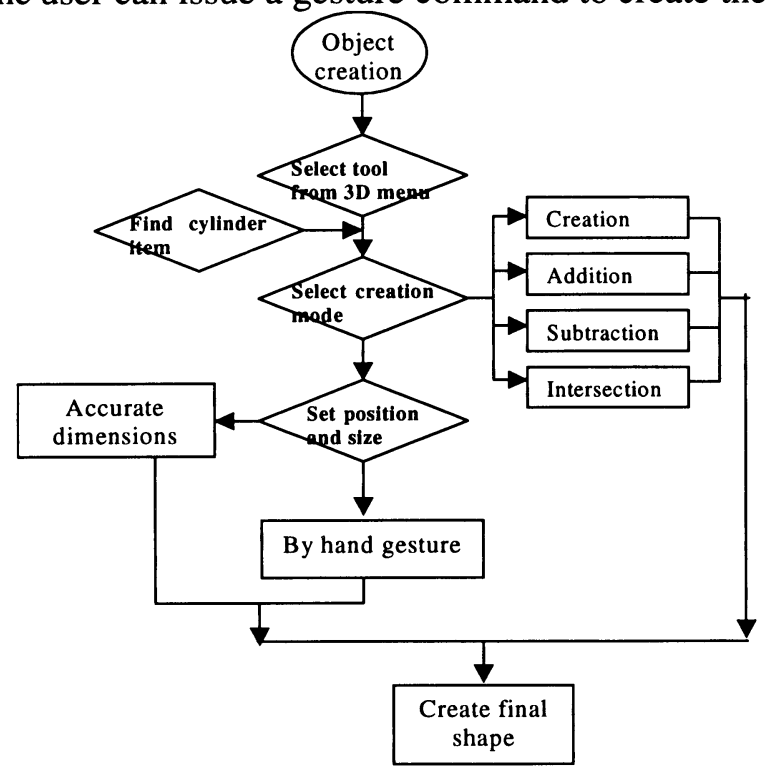

Figure 3 The process of creating a cylinder - an example for manipulation task

Object query: The object query task is to inquire the corresponding information about the selected entity. It can used to specify the dimension, the location and the relationships between the entities. Using object query option, the user can obtain a graphical representation of a model with its corresponding dimensions and expressions. The user can edit these parameters from the query information and update the model to reflect the changes that have been made. 


\subsection{Gesture interface design}

Gesture taxonomy: In the VR-based CAD system, we employ Caroline Hummels' classification [20] to categorize the gestures involved in the system. The gestures used in the system are considered as predefined symbolic commands for a given application in terms of the tasks the user is permitted to perform. We employ this type of gesture interface for the VRbased CAD system because it is easier to recognize a predefined hand posture instead of interpreting the infinite series of daily used gestures. In general, gestures involved in the VR-based CAD system are defined by very simple symbols so that they can be easily recognized. Moreover, the gestures can be divided into indicative gesture and manipulative gesture. The indicative gestures are used for creating and choosing menu item or icon. The manipulative gestures are used to guide the users to define, select, and manipulate the objects. The commands defined by the gestures are interpreted by the icons in the menu item or in the dialog box.

Gesture generation and recognition: In order to capture the meaning of the gesture, gesture generation has to be broken into two phases: analysis and recognition. Analysis is the process of converting the hand movements into a computational representation so as to produce a characteristic (feature) for the gesture. Recognition is a context-dependent process. It is a process to select appropriate data from the whole gesture library, which carries information comprehensive enough about the gesture instances, so that the system can decide if the conducted gesture has been accepted by the system and produce a gesture vocabulary. Generally, the process for gesture creation and recognition consists of three steps: data inputs, gesture feature extraction, and gesture vocabulary output.

Gesture interface: The gesture interface enables a user to give commands by making certain hand gestures. One-handed input gestures are used to provide a command context including menu operations, rigid solid manipulation and freeform surface definition and modification. Each of the gesture recognition systems works sequentially, receiving data sampled from an electronic hand glove device, analyzing the data, and sending them to an interpreter, which responds to user's gestures within an application context.

The main characteristic of the gesture driven interface is that the manipulation commands are pre-defined for a given application in terms of the tasks a user is permitted to perform. It is very easy to define our own set of gestures and to train the system users to explicitly recognize the gestures from this set. Our approach has the following advantages: i) the gestures do not need to be physically performed for the teaching procedure; ii) gestures can be easily defined by the system and used by people. 
3D graphics user interface: 3D graphics user interface elements involved in the VR-based CAD system consist of floating menus, 3D widgets, and gesture interface.

Floating menus: Because the menu is well accepted in the $2 \mathrm{D}$ community, we still employ the menu as one of the interface elements to provide a large amount of command choices. The floating menu displays two-dimensional menus in the three-dimensional world of VR. These menus are either text-based, describing the available choices with words, or graphics-based, using icons to convey the available choices. The floating menus include: feature based menu and freeform menu.

3D widgets: $3 \mathrm{D}$ widgets are objects in the virtual world that present an intuitive, direct manipulation interface to the user. Using $3 \mathrm{D}$ widgets as a user interface has many advantages. For example, the action of the "virtual hand", displayed in the virtual environment, directly conveys the action of the "real hand" in the "real world".

\section{GEOMETRIC MODELLING TOOLS}

Constructive technique: The constructive technique allows the user to assemble a representation of an object as a collection of features, through either adding or subtracting features from the model. It describes a product model into a context dependent feature-based representation. It is a process in which a model is constructed from a library of features, rather than geometric primitives. In this way, features are used as a basis for a number of design activities, including the design of components using standard shape features. The VR-based CAD system offers the user a fixed set of features to choose from. The form of a feature instance can be created on a geometric model by a procedure based on a given set of feature parameters.

According to constructive technique, the user should first extract a set of shape features from the product model, then convert them into a context dependent feature-based model. To convert the model into a context dependent feature-based model, it is necessary to interpret the feature information according to the feature classifications defined by the system. The description of the features in the VR-based CAD system can be divided into two general classes: protrusions and depressions. The protrusion features include solid primitives such as cube, cylinder, sphere, cone, tube, boss, pad, and swept features. The depression features include hole, slot, groove, and pocket.

Destructive techniques: In the destructive technique, feature can be considered as a cutting tool that allows the designer to subtract materials from the target object similar to a machining operation. The VR-based CAD 
system provides cutting tools for the user to cut a workpiece with virtual hand. The purpose of using destructive technique for solid modelling is to explore the feasibility of using the VR-based CAD system to sculpt an existing part by using a cutting tool. The simulation of sculpting is achieved by subtracting the tool's sweeping volume from the workpiece model. Three essential geometric entities involved in the simulation must be modeled, namely the part, the workpiece and the swept volumes created by the cutting tool. The model of the workpiece is dynamically changing as the swept volumes of the cutting tool are subtracted from it. The graphical display of the tool movement can be shown continuously.

Freeform shape design: The control point based mechanisms are widely used in the current CAD modelling systems to support the design of freeform surfaces. However, their mathematical representation often makes the interfaces unfriendly to the users. Our VR-based CAD system attempts to alleviate those limitations so as to assist the conceptual designer to create and deform freeform surfaces. The VR-based CAD system can allow the use of some non-control point based high-level shape operators to create and deform the freeform surfaces. The interactive sculpting technique allows the user to locally "sculpt" a 3D model. There are two kinds of manipulation tools in the VR-based CAD system: i) parameterization and ii) sculpting. Parameterization refers to the changing of the shape parameter of the surface in relation to the creating mode. Sculpting refers to the arbitrary deformation of the object shape. The system offers three types of sculpting tools: pointsbased, curve-based, and feature-based. The points-based deformation refers to the movement of points on a surface. Curve-based deformation refers to the movement of a curve that lies on a surface. The feature-based deformation refers to the addition of a feature surface to an existing surface.

\section{CONCLUSION}

This paper addresses the design of a VR-based CAD system for conceptual design. VR interfaces and geometric modelling techniques are applied to improve the intuitiveness of human computer interaction. From our work, it is considered that VR-based interfaces are more powerful and better matched to human sensory capabilities than those that only depend on traditional keyboard and mouse input methods. The VR-based CAD system is implemented as a desktop based operation system which inherits many user interface styles of Windows operating system, and hence allows the users who are familiar with Windows to use the system efficiently. 


\section{REFERENCE}

[1] Dani, T. H and Gadh, R., Creation of conceptual shape designs via virtual reality interface, CAD, Vol. 29, No. 8, pp. 555-563, 1997.

[2] Mine, M. R., ISAAC: a meta-CAD system for virtual environments, CAD, Vol. 29, No. 8, pp. 547-553, 1997.

[3] VENUS, http://sgvenus.cern.ch/VENUS/vr_project.html, 1999.

[4] Clemson's VR project: http://www.vr.clemson.edu/vr/, 1999.

[5] Chapin, W. L., Lacey, T. A. and Leifer, L., DesignSpace: a manual interaction environment of computer aided design, Human factors in computing systems, the ACM CHI'94 conference proceedings, Boston, Ma, April 1994.

[6] I-CARVE LAB, the University of Wisconsin, http://smartcad.me.wisc.edu/groups/virtual/virtual.html, 1999.

[7] Chu, C. P., Dani, T. H. and Gadh, R., Multi-sensory user interface for a virtual-realitybased computer-aided design system, CAD, Vol. 29, No. 10, pp. 709-725, 1997.

[8] Zeleznik, R. C., Herndon, K. and Hughes, J. F., SKETCH: An interface for sketching 3D scenes, Proceedings of SIGGRAPH '96.

[9] Forsberg, A. S., LaViola, J. J., Markosian, L. E. and Zeleznik, R. C., Seamless interaction in virtual reality, IEEE CG\&A, Vol. 17, No. 6, November, 1997.

[10] Zeleznik, R. C., Forsberg, A. S. and Strauss, P. S., Two pointer input for 3D interaction, Proceedings of 1997 Symposium on Interactive 3D Graphics, Providence, Rhode Island, April 27-30, 1997.

[11] Bloomenthal, M., Zeleznik, R. C., Fish, R., Holden, L. S., Forsberg, A. S., Riesenfeld, R., Cutts, M., Drake, S., Fuchs, H., and Cohen, E., Sketch-N-Make: automated machining of CAD sketches, Proceedings of the 1998 ASME 8th Computers In Engineering Conference, Atlanta, Georgia,1998.

[12] Forsberg, A. S., LaViola, J. J. and Zeleznik, R. C., ErgoDesk: A framework for two- and three-dimensional interaction at the ActiveDesk, Proceedings of the Second International Immersive Projection Technology Workshop, Ames, Iowa, May 11-12, 1998.

[13] Miller, T. and Zeleznik, R., The design of 3D haptic widgets, Proceedings of 1999 Symposium on Interactive 3D Graphics, ACM SIGGRAPH, 1999.

[14] Liang, J., JDCAD: a highly interactive 3D modelling system, Computer and Graphics, Vol. 18, No. 4, pp. 499-506, 1994.

[15] GVU Center Virtual Environments Group, http://www.cc.gatech.edu/gvu/virtual/CDS/, 1999.

[16] Kahaner, D., Japanese activities in virtual reality, IEEE CG\&A, pp. 75-78, January 1994.

[17] Nobel, R. A. and Clapworthy, G. J., Virtual Sculpting: A new approach to geometric modelling, Proceedings of the $2^{\text {nd }}$ UK VR-SIG Conference, pp. 66-79, December 1994.

[18] Trika, S. N., Banerjeer, P. and Kashyap, R. L., Virtual reality interfaces for feature-based computer-aided design systems, CAD, Vol. 29, No. 8, pp. 565-574, 1997.

[19] Gobel, M., Industrial application of VEs, IEEE CG\&A, pp. 10-13, January, 1996.

[20] Hummels, C., Smets, G. and Overbecke, K., An intuitive two-hand interface for computer supported product design, Proceedings of Interna-tional Gesture Workshop, Bielefeld, Germany, pp. 197-208, September 1997. 